\title{
Minimally Invasive Living Donor Hepatectomy
}

\author{
Talia B. Baker • Felicitas Koller • Juan Carlos Caicedo
}

Published online: 19 December 2013

(C) Springer International Publishing AG 2013

\begin{abstract}
Laparoscopic approaches to complex hepatobiliary cases are becoming more commonplace. As the practice of living donor liver transplants (LDLT) has evolved into an accepted alternative for certain patients as a source of donor organs, the application of laparoscopic techniques to these operations has also been introduced with an interest in improving donor outcomes and potential appeal of the donor procedure. The scope of laparoscopy ranges from the laparoscopically assisted left or right hepatic lobectomy ("hybrid") approach, to pure laparoscopic left lateral segments. Recently, robotic and purely laparoscopic approaches to right and left lobes have been reported by advanced minimally invasive surgeons with extensive experience in robotic and laparoscopic liver resection. Safety remains paramount to the performance of LDLT, and laparoscopic techniques must always be considered in light of optimal donor safety.
\end{abstract}

Keywords Minimally invasive $\cdot$ Laparoscopic $\cdot$ Liver transplant $\cdot$ Donation $\cdot$ Living donation $\cdot$ Hepatobiliary Hybrid approach · Pure laparoscopy · Safety · Laparoscopically assisted $\cdot$ Right lobectomy $\cdot$ Left lobectomy ·

T. B. Baker $(\bowtie) \cdot$ F. Koller $\cdot$ J. C. Caicedo

Comprehensive Transplant Center, Northwestern University,

Feinberg School of Medicine, 676 North St Claire Street Suite 1900,

Chicago, IL 60611, USA

e-mail: tabaker@nmh.org

F. Koller

e-mail: fkoller@nmh.org

J. C. Caicedo

e-mail: jcaiced@nmh.org

\section{J. C. Caicedo}

Northwestern Memorial Hospital, Children's Memorial Hospital, Northwestern University, Feinberg School of Medicine, 676 North St Claire Street Suite 1900, Chicago, IL 60611, USA
Left lateral sectionectomy · Donor hepatectomy · Open donor hepatectomy $\cdot$ Robotics $\cdot$ Minilaparotomy

\section{Introduction}

Each year, roughly 16,000 patients are listed for liver transplant, yet just over 6,000 receive transplants, and more than 2,000 on the waiting list die. It is imperative to find and improve ways to increase the number of liver grafts to meet demand and decrease mortality.

Living liver donation (LLD) is a viable alternative to liver transplantation from deceased donors. In light of critical organ shortages, LDLT can provide additional organs from a potentially unlimited source of healthy donors. Living donors provide excellent quality grafts with similar or even better shortterm graft function and long-term survival. This is true especially in children, as compared with whole and split liver transplant $[1,2]$. Currently, however, only about $4 \%$ of the liver recipients in the United States receive a living donor transplant [3].

The kidney transplant community responded to organ shortages with the successful use of a variety of minimally invasive approaches to living donor nephrectomy [4].The result of this evolution in technique has been an improved appeal and subsequen increase in the number of living kidney donors $[4,5]$.

The living liver donor community is certainly primed and ready for a similar strategy. Minimally invasive hepatobiliary techniques such as laparoscopic, hand-assisted laparoscopic, and robotic surgery have evolved over the past two decades to a point where their application in living donation is reasonable. These minimally invasive hepatobiliary procedures have many advantages over conventional open surgery including more rapid postoperative recovery, fewer pulmonary complications, and an excellent cosmetic outcome. Furthermore, the 
more recent developments in laparoscopic liver resection have shown the potential for a real reduction in morbidity as compared to standard open techniques $[6,7,8 \cdot 9]$. A move towards this type of innovative strategy to increase the number of liver grafts by making LLD safer and more appealing to potential donors, therefore, should be aggressively pursued.

Considering the magnitude and complexity of any donor hepatic surgery, the traditional approach has been an open donor surgery. For instance, the accepted approach for open donor right hepatectomy (ODRH) has been a bilateral subcostal incision with midline extension (Mercedes Incision) [10]. A subsequent modification to decrease the length of the incision was a right subcostal incision with midline extension (J-shape incision), avoiding the left subcostal extension [11]. The prospect of this large incisional scar may make some live liver donors reluctant to undergo the surgery due to concerns about self-image. The open approach is associated with significant wound complications, surgical pain, surgical time, recovery time, length of stay, and affects the quality of life of living donors [12-14]. Furthermore, despite open donor right hepatectomy (ODRH) being accepted, well described [15], and characterized as a relatively safe procedure, it is associated with a complication rate of up to $40 \%$ [14]. Efforts to introduce minimally invasive techniques to LLD, therefore, must be geared towards reducing this overall complication rate. Some of the numerous innovations to use minimally invasive techniques to improve upon open donor hepatectomies include the following:

- laparoscopic left lateral segmentectomy [1,2]

- laparoscopic hand-assisted right hepatectomy [13, 16]

- laparoscopic hand-assisted right hepatectomy using midline incision "Hybrid Method" [17, 18, 19•]

- laparoscopic hand-assisted right hepatectomy using a transverse or subcostal incision [13, 20]

- single port laparoscopic-assisted right hepatectomy using a right subcostal incision [20]

- right hepatectomy through upper midline laparotomy $[11,21]$

- right hepatectomy through an upper midline minilaparotomy with or without laparoscopic assistance [22]

- totally laparoscopic full left hepatectomy [23•]

- pure laparoscopic right hepatectomy [3]

- robot-assisted right hepatectomy [24•]

In this chapter, we will describe the advantages and disadvantages of some of the important variations of these minimally invasive approaches to donor hepatectomy. Table 1 provides a descriptive overview and comparison of these approaches. It is important to remember as each is presented that they must be considered with attention to their efficacy, feasibility, and ability to provide a safe and appealing procedure for any potential donor.

\section{Laparoscopically Assisted Right Hepatic Lobectomy ("Hybrid Technique")}

In response to the continued significant interest in laparoscopic liver surgery as it may apply to living donation, but also with an eye towards developing a procedure which may be more widely applicable to all donor surgeons, we first performed a laparoscopically assisted donor right hepatectomy (LADRH) at Northwestern (Chicago, IL) in 2006 [17]. In our first and other publications $[6,9,17,18,19 \bullet]$, we and others have demonstrated the safety and efficacy of laparoscopically assisted hepatectomy for the resection of hepatic tumors with reduced blood loss, shorter operative times, and decreased postoperative hospital stays, as well as its applicability to living donation $[17,18,19 \bullet]$.

The approach typically uses the "hybrid method", a laparoscopically assisted method for liver resection. The technique represents a hybrid between the minimally invasive and open techniques. The hybrid technique abrogates the need for standard subcostal incisions in most liver resections and, therefore, is consistent with the principles of minimally invasive surgery. The incisions required are identical to those used for a hand-assisted laparoscopic procedure. This surgical approach can be performed by surgeons with minimal laparoscopic skills, does not require laparoscopic techniques and devices for either dissection of the hepatic hilum or transection of the liver parenchyma, and thus allows surgeons to adhere to more familiar, open techniques that may be safer in their hands.

\section{Technique for LADRH}

As this is the technique that we at Northwestern have espoused, we will herein specifically describe the technical aspects of the approach as we have previously reported. [17] The donor is placed in the supine position, arms tucked at the sides with large bore IVs, a right radial arterial a-line, and a Foley catheter, then placed under general anesthesia. Pneumoperitoneum is established through a $12 \mathrm{~mm}$ port placed in the upper midline at or above the umbilicus, using Hassan Technique for safety. The abdomen is explored using a 30degree, $10 \mathrm{~mm}$ laparoscope to allow for optimal optics. Once the liver is visualized and there are no obvious contraindications to proceeding with donation, one additional $5 \mathrm{~mm}$ port is placed in the right midclavicular line, and a $5 \mathrm{~cm}$ subxiphoid midline incision is created for hand assistance during right lobe mobilization using a GelPort hand port device (Applied Medical, Rancho Santa Margarita, CA, USA) (Fig. 1a). This configuration allows the operating surgeon, who is standing on the left side of the patient, to use the hand port for graft manipulation with simultaneous use of the midclavicular line port for dissection. LigaSure (Valley Lab, Boulder CO, USA) is used to divide the ligamentum teres, falciform ligament, and 


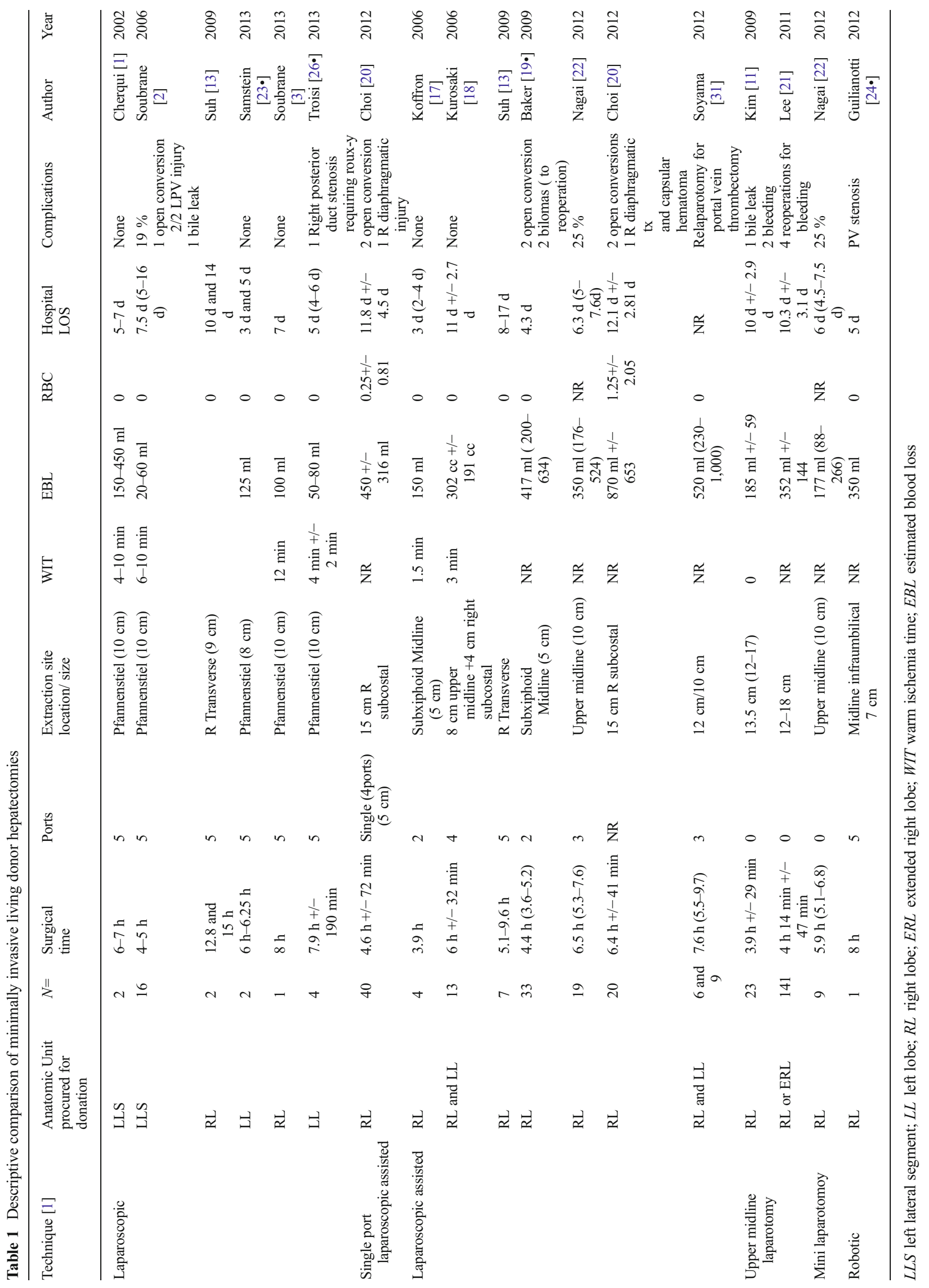


right triangular ligaments. The hepatic bare area is completely mobilized, the right lobe elevated exposing the length of the retrohepatic inferior vena cava (IVC). The posterior vena cava ligament and the short hepatic veins are divided using LigaSure to separate the IVC and the right lobe, allowing visualization of the right hepatic vein (RHV) as it enters the IVC. At this point the hand port and all other laparoscopic devices are removed; the upper midline hand assist port incision is extended to include the camera port, and a liver transplant retractor is placed. Ensuring that no nerve damage is caused by excessive traction on the brachial plexus is accomplished by carefully watching the waveform in the right radial arterial line to ensure that there is no dampening with progressive retraction. Under direct visualization, the right hepatic vein is encircled with an umbilical tape creating a "hanging maneuver" (previously described) [25]. An open cholecystectomy is then performed and the cystic duct is cannulated in preparation for a cholangiogram. Once cholangiography confirms the presence of suitable anatomy, the right hepatic duct is isolated, and using fluoroscopic cholangiographic guidance, the right hepatic duct (RHD) is transected sharply, taking care to leave an appropriate stump of RHD on the donor hepatic duct confluence, which is subsequently oversewn with 7-0 monofilament, nonabsorbable suture. The right hepatic artery (RHA) and right portal vein (RPV) branches are completely dissected with extreme care along an appropriate length. It should be noted that no inflow occlusion is used at any point during the case. A "tape switch maneuver" whereby the umbilical tape is transferred superior to the RPV/RHA confluence to protect these vessels during liver transection, is then performed. The liver parenchyma is then divided at Cantlie's line using the hanging umbilical tape to bring the plane of dissection up into the midline incision. The parenchymal transection is achieved in an inferior/ anterior to superior/posterior plane using a combination of Helix Hydrojet (ERBE USA incorporated Surgical Systems, Marietta GA, USA) and the LigaSure device. The middle hepatic vein tributaries from segments five and eight are ligated or preserved for reimplantation at the surgeon's discretion (usually when $>5 \mathrm{~mm}$ ). Once the hemilivers are completely separated and the recipient operative team notified, the RHA is ligated and sharply divided, allowing back bleeding from the graft side. Immediately, the RPV is stapled but not divided using a $30 \mathrm{~mm}$ TA vascular stapling device (US Surgical, Norwalk CT USA). The right hepatic lobe is then retracted laterally and the RHV stapled and divided at the IVC using a laparoscopic endovascular GIA stapler (US Surgical). The RPV is then transected sharply on the graft side using scissors. The right lobe is decompressed by allowing back bleeding of the graft through the RPV and the right lobe is extracted through the upper midline incision. The RHA stump is oversewn with 7-0 nonabsorbable monofilament, and the RPV and RHV stumps are inspected for hemostasis.
Upon completion, a cholangiogram is performed to confirm the integrity of the remaining biliary system and to ensure that there are no bile leaks. The falciform ligament is then reconstructed to resuspend the left hepatic lobe from the anterior abdominal wall. A closed suction drain is then placed through the previously situated $5 \mathrm{~mm}$ right midclavicular line port site to service the remnant liver cut surface; this is secured to the skin with a 3-0 nonabsorbable suture. The extraction incision is then closed in layers.

On the back table, the vascular staple lines on the RHV are excised and the right lobe is flushed with ice cold Custodial HTK solution (Odyssey Pharmaceuticals, East Hanover NJ, USA). Ideally this is done within $90 \mathrm{sec}$ of vascular exclusion.

\section{Upper Midline ("Mini Laparotomy Approach")}

In response to the proliferation of laparoscopic and laparoscopically assisted approaches to living liver donation, many programs have reported on a mini laparotomy approach without laparoscopic assistance in appropriately selected candidates. Specifically in patients with low BMI $(<25$ or less) and small anterior/posterior diameter, certain surgeons have described an upper midline approach through which they are able to accomplish complete mobilization of the right lobe from its retroperitoneal attachments and off the right side of the vena cava [22]. They then proceed with hilar dissection and transection of the liver using a hanging maneuver similar to what is described in the laparoscopically assisted approach. The advantages of this are obviously the lack of cost and expertise required for laparoscopy, but this approach is limited in applicability to a very specific body habitus in the prospective donor.

\section{Purely Laparoscopic Approaches}

\section{Left Lateral Segmentectomy}

Laparoscopic approaches to living donation were initially described for removing a left lateral segment graft for transplantation into a child $[1,2]$. Dr Cherqui's team initially demonstrated the feasibility of this laparoscopic living donor hepatectomy of the left lateral segment [1].

The procedure has been described with the patient under general anesthesia in the supine position with their legs apart. Five trocars were used. The left lateral segment was mobilized by dividing the falciform and left triangular ligaments; vascular structures were dissected and encircled. The liver transection was performed with harmonic scalpel (Ultracision, Ethicon, USA) and an ultrasonic dissector (Dissectron, Satelec, France) without vascular clamping. The bleeding was controlled with bipolar electrocoagulation and clips. A 
$10 \mathrm{~cm}$ suprapubic incision was made to introduce a $15 \mathrm{~mm}$ trocar to introduce a large specimen bag to retrieve the liver.

Laparoscopically Full Lobectomy for Donation

Recently, multiple case reports have been published detailing purely laparoscopic approaches to hepatic lobectomy [3, 23•, $26 \cdot]$. The procedures were all performed with patients in the supine position with legs adducted to 45 degrees while under general anesthesia. Five ports were used in all cases, but their configurations were variable (Fig. 1b).

In the right lobectomy [3] the cystic artery and duct were clipped and ligated. Then the pedicle was opened, and vascular structures were dissected free and taped. Cholecystectomy was performed, and the round and falciform ligaments were cut. The right part of segment I was mobilized to dissect the anterior aspect of the inferior vena cava. The vascular inflow was briefly clamped to demarcate the liver. The capsule was divided with a harmonic scalpel and the parenchyma with an ultrasonic dissector, bipolar coagulation, and clips. When the right bile duct level was reached, intra-operative cholangiography was performed via the cystic duct to mark the level of biliary transection. The liver section was then completed and the right hepatic vein was isolated. The right liver was mobilized by freeing the right triangular ligament. The right hepatic artery was closed with a secured clip and the right portal and hepatic veins were divided.

The left lobectomies began with mobilization of the left lobe of the liver followed by division of the cystic artery and duct [23•]. The left hepatic and left portal veins were dissected and encircled with umbilical tapes. The left bile duct was transected behind the quadrate lobe. The common trunk of the left and middle hepatic vein was encircled by tunneling between the trunk and the IVC above the caudate lobe. The liver was demarcated with transient clamping of inflow and the capsule scored with monopolar cautery. The transection of the liver was performed using an alternating combination of laparoscopic ultrasonic aspirator (CUSATM Excel, Valleylab Corp, Boulder, CO) and LigaSure VTM (Valleylab, Boulder, $\mathrm{CO}$ ). To complete the transaction, a hanging maneuver was used. A unilateral linear stapler (30 mm Endo TA; Covidien, Mansfield, MA) was used to seal the proximal left artery and a second firing for the left portal vein. The hepatic artery and portal vein were then divided with scissors. The common LHV/MHV trunk was transected using a vascular endostapler (endoGIA 45-2.5, Covidien) and the graft was removed through a Pfannenstiel incision. Troisi's group performs the procedure slightly differently with an earlier cholecystectomy and administration of heparin prior to division of the vascular structures [26•].

The advantages of a purely laparoscopic approach are the lack of an upper midline incision and the rapid recovery to normal function. As this technique requires significant experience with advanced laparoscopic skills, however, only surgeons very well versed in the art of advanced laparoscopic techniques can safely perform this operation, limiting its applicability.

\section{Robotically Assisted Donor Hepatectomy}

An increased interest in performing complex hepatobiliary cases using the robot led naturally to consideration of its use in living donor hepatectomy. The first report of a robotassisted right lobe donor hepatectomy was published by the University of Illinois at Chicago [24•]. Dr Giulianotti's group already had an extensive experience in robotic surgery including hepatobiliary cases using the Da Vinci Robotic Surgical System. They demonstrated that this type of approach to living liver donation is feasible. The main limitation to this technique, however, is the institutional and surgeon-specific experience in and cost of this type of technology that may limit its widespread use in most of the transplant centers. They emphasized that only experienced robotic surgical teams should consider attempting this type of procedure.

Four trocars were used for robotic and laparoscopic instruments and a $7 \mathrm{~cm}$ sub-umbilical midline laparotomy for hand assistance (Fig. 1c). Robotic Harmonic scalpel was used for the transection of the parenchyma, and no inflow clamping (Pringle) was used at anytime. The right lobe was extracted through the sub-umbilical midline laparotomy. The length of the procedure was $8 \mathrm{~h}$ with blood loss of $350 \mathrm{ml}$. The donor did not require any blood transfusion. The donor's postoperative course was uneventful, and he was discharged home on the fifth postoperative day. Six months post donation, the donor developed a short stenosis of the main trunk of the donor portal vein that was dilated through a percutaneous hepatic approach.

Some of the potential advantages of the robotic procedure are a stable magnified field, 3D vision, and enhanced instrument articulation to facilitate dissection of the biliovascular structures. The robot also offers enhanced ease in suture ligation of hepatic parenchymal bleeding points. Certainly, however, the disadvantages include the institutional expense of the robot as well as the extra time and resources required for its proper execution. Furthermore, application of this technique will be limited to those surgeons already facile in the use of the robot for complex hepatobiliary cases.

\section{Donor Safety and Outcomes: Potential Benefits and Future Applications of Laparoscopic Approaches}

Ultimately, the soundness of any technical innovation in living donation is determined by its ability to enhance donor 
Fig. 1 Novel individual approaches to minimally invasive living donor hepatectomy: Port Placements. a Schematic diagram of port placement for hybrid method lobectomy. Numbers are diameters of trocars $(\mathrm{mm})$ (with permission from: Koffron AJ, Kung R, Baker T, Fryer J, Clark L, Abecassis M. Laparoscopicassisted right lobe donor hepatectomy. American Journal of Transplantation : official journal of the American Society of Transplantation and the American Society of Transplant Surgeons. Oct 2006; 6(10):25222525) [17]. b Schematic diagram of port placement for totally laparoscopic right lobectomy. Numbers are diameters of trocars $(\mathrm{mm})$ (with permission from: Soubrane O, Perdigao Cotta F, Scatton O. Pure laparoscopic right hepatectomy in a living donor. American journal of transplantation: official journal of the American Society of

Transplantation and the American Society of Transplant Surgeons. Sep 2013; 13(9):2467-2471) [3]. c Schematic diagram of port placement for robotic donor hepatectomy, the extraction site is not shown (with permission from: Giulianotti PC, Tzvetanov I, Jeon $\mathrm{H}$, et al. Robot-assisted right lobe donor hepatectomy. Transplant International: official journal of the European Society for Organ Transplantation. Jan 2012;25(1): e5-9) [24•] a
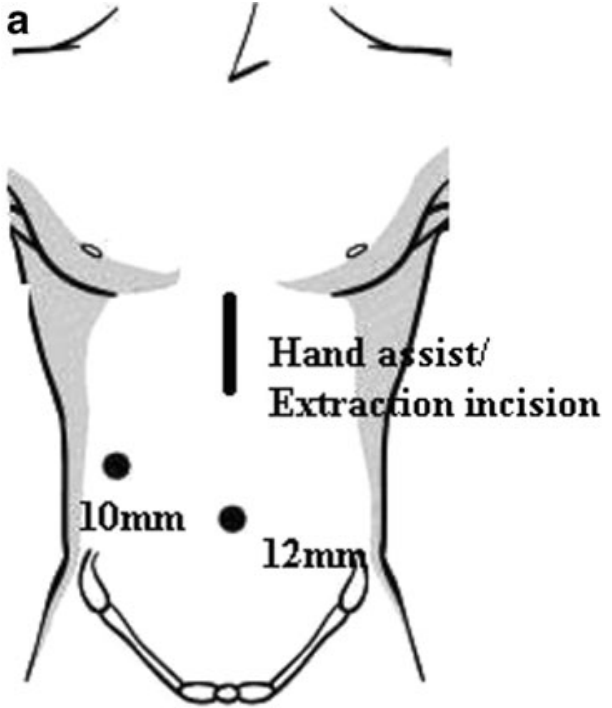

b<smiles>C[C]CCCC</smiles>

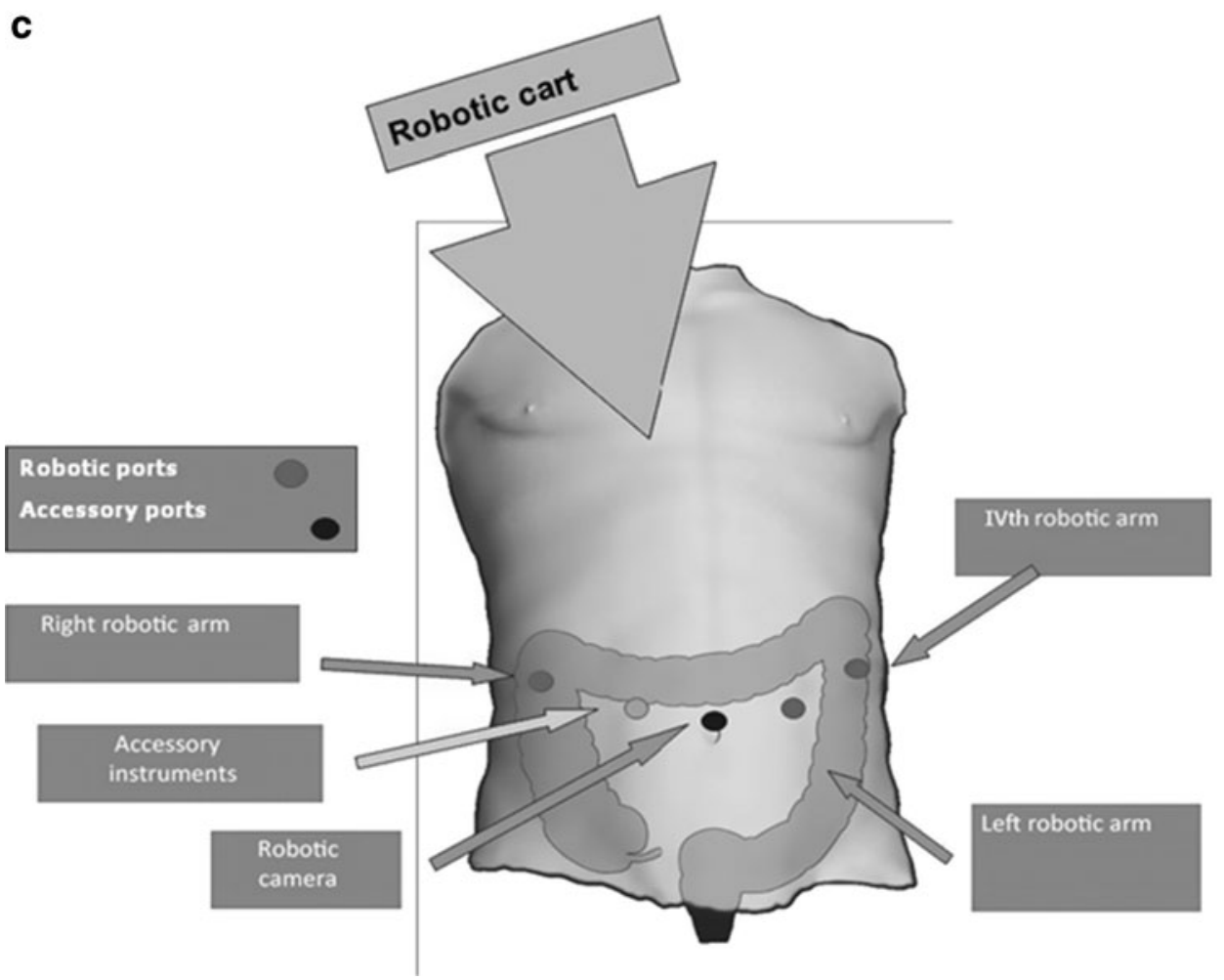

outcomes and have equivalent or improved safety as compared to the traditional open techniques. In LLD, complications are largely determined by physiological condition of the donor before and after donation, the amount of liver removed, and potentially intraoperative blood loss $[2,3]$. The types of complications in LLD are often technical such as bleeding, bile leaks or hernia as well as complications of post-operative recovery such as ileus, pneumonia, wound infection, or DVT. The Clavien classification is a standardized way to compare complications between procedures and transplant centers. In the most comprehensive review to date, the $\mathrm{A} 2 \mathrm{ALL}$ consortium recently reported that the most common complications of living liver donation were infections, pleural effusion, bile leak, and hernia, with an overall complication rate of $\sim 40 \%$ [27••].

When considering laparoscopic-assisted and purely laparoscopic LLD, it is clear that laparoscopy does not change the physiologic condition of the donor before surgery. However, laparoscopy does have the potential to reduce some technical complications as well as complications of post-operative recovery. For example, Baker and co-workers found that operative time was shorter for laparoscopic-assisted right donor 
hepatectomy LADRH ( $265 \pm 48$ minutes) than open right donor hepatectomy ODRH $(316 \pm 61$ minutes; $\mathrm{P}<.001)$ [19•]. Reduced operative time as well as minimized incision may lead to reduced pulmonary complications and earlier mobilization and leave the donor in better physiologic shape after operation.

An intriguing aspect of both laparoscopic-assisted procedures and totally laparoscopic liver resection may be reduced blood loss compared to open procedures. Baker and co-workers found that estimated blood loss was lower after LADRH, although this difference is not statistically significant (LADRH $417 \pm 217 \mathrm{ml}$; ODRH $550 \pm 305 \mathrm{ml}, \mathrm{P}=.10$ ) [19•]. Additional centers have found reduced blood loss with totally laparoscopic approaches [18]. Soubrane's group had a blood loss of an average of $120 \mathrm{~mL}$ in a series of totally laparoscopic liver resections that included both for-cause resections and living donor livers. They found this was lower than what has been traditionally reported during open liver resections [28]. Possible explanations for this include the 30-degree reverse Trendelenburg position reducing hepatic backflow, more effective hemostasis on the cut surface due to lap-enhanced vision and possibly the effects of pneumoperitoneum on cut surface bleeding [28]. The possibility of reduced blood loss and need for transfusions is important, as blood transfusion is associated with a significantly higher risk of complication, and specifically of bile leaks and infection. In the A2ALL consortium study, each unit of transfused blood was associated with a $38 \%$ to $55 \%$ higher risk of complication $[27 \cdot \bullet]$.

Current work does not demonstrate a decreased length of stay (LOS) or earlier return to normal activity, but this has not been widely studied. The ability to show a significant difference in LOS between laparoscopic, hybrid, and open procedures may be challenging, as LOS may be more related to institutional discharge practices as well as national differences in LOS as related to healthcare cost $[2,19 \bullet]$.

Importantly, both Akoad and Mulligan stress that the case reports of totally laparoscopic and case series of laparoscopicassisted liver donation are not procedures that the reporting centers just "started" de novo. Rather, they are a reflection of accumulated expertise in both laparoscopy and liver resection $[29 \bullet \bullet, 30 \bullet$. Furthermore, Mulligan raises the issue of control of major vascular complications as being a serious safety concern in any hepatobiliary laparoscopic procedure.

\section{Summary and Conclusion}

Minimally invasive procedures using laparoscopic techniques for liver graft procurement have the potential to improve donor safety and increase the number of willing living donors and decrease waiting list mortality. In this regard, the liver transplant community can learn from the experience of kidney transplantation where the use of laparoscopic techniques has become part of the standard of care for kidney donation.

Minimally invasive approaches to living donation can vary from pure laparoscopy, laparoscopically assisted ("hybrid") techniques, mini laparotomies, and robotically assisted procedures. All have been developed to avoid the painful subcostal incision, allowing faster recovery and to minimize morbidity associated with the incision. All have also been developed as a means to improve acceptability and appeal of the procedure to potential donors. All of these techniques certainly warrant further evaluation for living liver donation.

For left lateral segmentectomies, either mini laparotomy through a modified and limited upper midline incision or pure laparoscopic approaches are certainly reasonable. The pure laparoscopic approach requires significant advanced laparoscopic skills, limiting its applicability. Furthermore, an incision must ultimately be made (albeit in the suprapubic position) for graft removal, bringing into question the ultimate benefit for a perhaps less safe approach.

Right lobe donors can be approached in a number of minimally invasive manners as described prior. Robotic assistance requires significant institutional and surgeon-based expertise and is associated with significant costs. The length of the procedure also will likely prove to be limiting for most surgeons. It will likely be difficult to define significant advantage to this approach. The mini laparotomy approach is attractive in that it allows the advantage of a limited incision and removes the added cost and required expertise associated with laparoscopic assistance. The donor pool to which this approach is applicable is very limited.

In our experience we believe that the "hybrid" approach to right lobe living donor hepatectomy has significant advantages and emerges as the optimal minimally invasive approach. First and foremost, it offers a minimal upper midline incision, addressing the primary objective of the development of novel approaches. Furthermore, the limited nature of the laparoscopic mobilization allows most surgeons to perform the procedure after a minimal learning curve. The majority of the hilar dissection and parenchymal dissection is done under direct visualization through the midline incision with assistance of the hanging maneuver. The tenet of surgical innovation with a commitment to optimal donor safety is therefore met with the application of this technique.

\section{Compliance with Ethics Guidelines}

Conflict of Interest Talia B. Baker, Felicitas Koller, and Juan Carlos Caicedo declare that they have no conflict of interest.

Human and Animal Rights and Informed Consent This article does not contain any studies with human or animal subjects performed by any of the authors. 


\section{References}

\section{Papers of particular interest, published recently, have been highlighted as \\ - Of importance \\ •- Of major importance}

1. Cherqui D, Soubrane O, Husson E, et al. Laparoscopic living donor hepatectomy for liver transplantation in children. Lancet. 2002;359(9304):392-6.

2. Soubrane O, Cherqui D, Scatton O, et al. Laparoscopic left lateral sectionectomy in living donors: safety and reproducibility of the technique in a single center. Ann Surg. 2006;244(5):815-20.

3. Soubrane O, Perdigao Cotta F, Scatton O. Pure laparoscopic right hepatectomy in a living donor. Am J Transplant Off J Am Soc Transplant Am Soc Transpl Surg. 2013;13(9):2467-71.

4. Schweitzer EJ, Wilson J, Jacobs S, et al. Increased rates of donation with laparoscopic donor nephrectomy. Ann Surg. 2000;232(3): 392-400.

5. Jacobs SC, Cho E, Dunkin BJ, et al. Laparoscopic live donor nephrectomy: the University of Maryland 3-year experience. J Urol. 2000;164(5):1494-9.

6. Buell JF, Koffron AJ, Thomas MJ, Rudich S, Abecassis M, Woodle ES. Laparoscopic liver resection. J Am Coll Surg. 2005;200(3):472-80.

7. Lesurtel M, Cherqui D, Laurent A, Tayar C, Fagniez PL. Laparoscopic versus open left lateral hepatic lobectomy: a casecontrol study. J Am Coll Surg. 2003;196(2):236-42.

8. Rau HG, Buttler E, Meyer G, Schardey HM, Schildberg FW. Laparoscopic liver resection compared with conventional partial hepatectomy-a prospective analysis. Hepatogastroenterology. 1998;45(24):2333-8. This is a landmark paper which underscores the feasibility and efficacy of laparoscopic approaches to laparoscopic surgery.

9. Buell JF, Cherqui D, Geller DA, et al. The international position on laparoscopic liver surgery: The Louisville Statement, 2008. Ann Surg. 2009;250(5):825-30.

10. Marcos A, Fisher RA, Ham JM, et al. Right lobe living donor liver transplantation. Transplantation. 1999;68(6):798-803.

11. Kim SH, Cho SY, Lee KW, Park SJ, Han SS. Upper midline incision for living donor right hepatectomy. Liver Transpl Publ Am Assoc Study Liver Dis Int Liver Transplant Soc. 2009;15(2):193-8.

12. Parikh ND, Ladner D, Abecassis M, Butt Z. Quality of life for donors after living donor liver transplantation: a review of the literature. Liver Transpl Publ Am Assoc Study Liver Dis Int Liver Transplant Soc. 2010;16(12):1352-8.

13. Suh KS, Yi NJ, Kim T, et al. Laparoscopy-assisted donor right hepatectomy using a hand port system preserving the middle hepatic vein branches. World J Surg. 2009;33(3):526-33.

14. Trotter JF, Talamantes M, McClure M, et al. Right hepatic lobe donation for living donor liver transplantation: impact on donor quality of life. Liver Transpl Publ Am Assoc Study Liver Dis Int Liver Transplant Soc. 2001;7(6):485-93.

15. Suh KS, Kim SH, Kim SB, Lee HJ, Lee KU. Safety of right lobectomy in living donor liver transplantation. Liver Transpl Publ Am Assoc Study Liver Dis Int Liver Transplant Soc. 2002;8(10):910-5.

16. Suh KS, Yi NJ, Kim J, et al. Laparoscopic hepatectomy for a modified right graft in adult-to-adult living donor liver transplantation. Transplant Proc. 2008;40(10):3529-31.

17. Koffron AJ, Kung R, Baker T, Fryer J, Clark L, Abecassis M. Laparoscopic-assisted right lobe donor hepatectomy. Am J Transplant Off J Am Soc Transplant Am Soc Transpl Surg. 2006;6(10):2522-5.
18. Kurosaki I, Yamamoto S, Kitami C, et al. Video-assisted living donor hemihepatectomy through a 12-cm incision for adult-to-adult liver transplantation. Surgery. 2006;139(5):695-703.

19. Baker TB, Jay CL, Ladner DP, et al. Laparoscopy-assisted and open living donor right hepatectomy: a comparative study of outcomes. Surgery. 2009;146(4):817-23. discussion 823-815. First longitudinal report of a large series of minimally invasive approaches for living donation establishing the feasibility and efficacy of the procedure.

20. Choi HJ, You YK, Na GH, Hong TH, Shetty GS, Kim DG. Singleport laparoscopy-assisted donor right hepatectomy in living donor liver transplantation: sensible approach or unnecessary hindrance? Transplant Proc. 2012;44(2):347-52.

21. Lee KW, Kim SH, Han SS, et al. Use of an upper midline incision for living donor partial hepatectomy: a series of 143 consecutive cases. Liver Transpl Publ Am Assoc Study Liver Dis Int Liver Transplant Soc. 2011;17(8):969-75.

22. Nagai S, Brown L, Yoshida A, Kim D, Kazimi M, Abouljoud MS. Mini-incision right hepatic lobectomy with or without laparoscopic assistance for living donor hepatectomy. Liver Transpl Publ Am Assoc Study Liver Dis Int Liver Transplant Soc. 2012;18(10): 1188-97.

23. Samstein B, Cherqui D, Rotellar F, et al. Totally laparoscopic full left hepatectomy for living donor liver transplantation in adolescents and adults. Am J Transplant Off J Am Soc Transplant Am Soc Transpl Surg. 2013;13(9):2462-6. Landmark technical report of totally laparoscopic approach to living donor hepatectomy.

24. Giulianotti PC, Tzvetanov I, Jeon H, et al. Robot-assisted right lobe donor hepatectomy. Transpl Int Off J Eur Soc Organ Transplant. 2012;25(1):e5-9. First report of a robotically assisted living donor right hepatectomy.

25. Belghiti J, Guevara OA, Noun R, Saldinger PF, Kianmanesh R. Liver hanging maneuver: a safe approach to right hepatectomy without liver mobilization. J Am Coll Surg. 2001;193(1):109-11.

26. Troisi RI, Wojcicki M, Tomassini F, et al. Pure laparoscopic full-left living donor hepatectomy for calculated small-for-size LDLT in adults: proof of concept. Am J Transplant Off J Am Soc Transplant Am Soc Transpl Surg. 2013;13(9):2472-8. Important report which compliments that of Samstein et al 22demonstrating potential feasibility of totally laparoscopic approaches to living liver donation .

27.• Abecassis MM, Fisher RA, Olthoff KM, et al. Complications of living donor hepatic lobectomy-a comprehensive report. Am J Transplant Off J Am Soc Transplant Am Soc Transpl Surg. 2012;12(5):1208-17. Publication of the A2ALL consortium of North American living donor liver transplant centers defining the expected/acceptable morbidity and mortality associated with the procedure.

28. Costi R, Scatton O, Haddad L, et al. Lessons learned from the first 100 laparoscopic liver resections: not delaying conversion may allow reduced blood loss and operative time. J Laparoendosc Adv Surg Tech. 2012;22(5):6.

29.• Akoad ME, Pomfret EA. Laparoscopic live donor hepatectomy: random mutation or stepwise evolution? Am J Transplant Off J Am Soc Transplant Am Soc Transpl Surg. 2013;13(9):2243-4. Excellent editorial responding to the introduction of the idea of purely laparoscopic approaches to living liver donation with a focus on donor safety and emphasizing the caution with which transplant centers should consider this approach.

30.• Mulligan DC. Living donor safety during the performance of hepatectomy. Liver Transpl. 2012;18(10):1134-5. A cautionary editorial reflecting on the blossoming interest in minimally invasive approaches to living donor hepatecomy with an emphasis on the importance of surgeon specific experience with advanced laparoscopic skills prior to considering this endeavor.

31. Soyama A, Takatsuki M, Hidaka M, et al. Standardized less invasive living donor hemihepatectomy using the hybrid method through a short upper midline incision. Transplant Proc. 2012;44(2):353-5. 